VDI

K

Spritzgießen 2020 
KUNSTSTOFFTECHNIK

\section{Spritzgießen 2020}

Herausgeber: VDI Wissensforum GmbH 


\section{Bibliographische Information der Deutschen Bibliothek}

Die Deutsche Bibliothek verzeichnet diese Publikation in der Deutschen Nationalbibliographie; detaillierte bibliographische Daten sind im Internet unter www.dnb.de abrufbar.

\section{Bibliographic information published by the Deutsche Bibliothek}

(German National Library)

The Deutsche Bibliothek lists this publication in the Deutsche Nationalbibliographie (German National Bibliography); detailed bibliographic data is available via Internet at www.dnb.de.

\section{(c) VDI Verlag GmbH · Düsseldorf 2020}

Alle Rechte, auch das des auszugweisen Nachdruckes, der auszugweisen oder vollständigen photomechanischen Wiedergabe (Photokopie, Mikrokopie) und das der Übersetzung, vorbehalten.

Printed in Germany

ISBN 978-3-18-234355-4 
VDI-Programmausschuss Spritzgießtechnik

Dipl.-Ing. Roland Dörner, Tente-Rollen-GmbH, Wermelskirchen Dipl.-Ing. Bernhard Hoster, GIRA Giersiepen GmbH \& Co. KG, Radevormwald

Dipl.-Ing. Kerstin Krallmann, Erwin Quarder Werkzeugtechnik GmbH \& Co. KG, Espelkamp

Prof. Dr.-Ing. Karl Kuhmann, Evonik Resource Efficiency $\mathrm{GmbH}$, Marl Dipl.-Ing. Norbert Nobbe, Pöppelmann Holding GmbH \& Co. KG, Lohne

Dipl.-Ing. Guido Peters, gwk Gesellschaft Wärme Kältetechnik mbH, Meinerzhagen

Dipl.-Ing. Univ. Steffen Reuter, Kunststoff Schwanden AG, Schwanden (Schweiz)

Dr. Marco Wacker, Uvex Arbeitsschutz GmbH, Fürth

Dr.-Ing. Thomas Walther, ARBURG GmbH \& Co. KG, Loßburg

Dr.-Ing. Martin Wanders, Lanxess Deutschland GmbH, Dormagen

Dipl.-Ing. Martin Würtele, KraussMaffei Technologies $\mathrm{GmbH}$, München (Vorsitz) 
Inhalt

\section{Plenarvorträge}

Kreislauftechnik von Kunststoffen - Rückblick K-Messe, was war neu Prof. Dr.-Ing. H. J. Endres, Institut für Kunststoff- und Kreislauftechnik IKK, Leibniz Universität Hannover

Mikroplastik: Quellen, Mengen und Möglichkeiten der Reduzierung L. Hamann, J. Bertling, R. Bertling, T. Weber, Fraunhofer-Institut für Umwelt-, Sicherheits- und Energietechnik UMSICHT, Oberhausen

Vollautomatische modellgestützte Einrichtung von Spritzgießprozessen Unterstützung des Einrichters durch vollvernetzte Produktionszellen

S. Wurzbacher, M.Sc., Dipl.-Ing. M. Schmitz, Y. Lockner, M.Sc.,

B. Liu, M.Sc., Dr.-Ing. M. Röbig, Prof. Dr.-Ing. C. Hopmann, Institut für Kunststoffverarbeitung (IKV) an der RWTH Aachen, Aachen

Closed-Loop in der Nachhaltigkeit - Der Lebenslauf einer Filterkartusche Prof. Dr.-Ing. T. Schröder, L. Rieming, Institut für Kunststofftechnik Darmstadt (ikd), Darmstadt

Ganzheitlicher Einsatz von digitalen Methoden im Freigabeprozess S. Heienbrock, M.Sc., Prof. Dr.-Ing. W. Guth, Hochschule Esslingen, Labor für Kunststofftechnik, Esslingen

\section{Nachhaltiger Materialeinsatz}

Umsetzung vom physikalischen Schäumverfahren in der Serie Dipl.-Ing. (FH) J. Götzelmann, M. Benitez, Magna Exteriors GmbH, D-Sailauf; Dr. Pavel Petera, Magna Exteriors (Bohemia) s.r.o., CZ-Liberec

Hohlprofil-Hybridtechnik - Intelligenter Materialeinsatz für Strukturbauteile in der automobilen Großserie Dr.-Ing. M. Theunissen, B. Koch, Dr.-Ing. M. Wanders, LANXESS Deutschland $\mathrm{GmbH}$, Köln

Hochleistungspolymere für intelligente und funktionelle Bauteile Nachhaltiger Materialeinsatz

Dipl.-Ing. F. Lorenz, Prof. Dr.-Ing. K. Kuhmann, Evonik Resource Efficiency, Marl 
Qualitätsgesicherte Regranulate für hochwertige Spritzgussprodukte Dipl. Kauf. T. Kriele MBA, B.Sc. Wirt.-Ing. M. Kiffmeyer,

geba Kunststoffcompounds $\mathrm{GmbH}$, Ennigerloh

Sandwich-Spritzgießen - ein Beitrag zur besseren Verwertung von

Recycling-Kunststoffen

Dr.-Ing. V. Reichert, A\&E Produktionstechnik GmbH, Dresden;

A. Handschke, KraussMaffei Technologies $\mathrm{GmbH}$, München

\section{Ressourceneffiziente Prozessführung}

Virtuelle Produktionsassistenz im Spritzgießprozess

Dr.-Ing. K. Saul, SHS plus GmbH, Dinslaken

KI-System für robuste Produkte und stabile Prozesse am Praxisbeispiel

Dipl.-Ing. F. Thurner, Contech Software \& Engineering GmbH, Fürstenfeldbruck

Servo-angetriebene Heißkanalsysteme - Erweiterte Prozessmöglichkeiten und signifikante Qualitätsverbesserung

Dipl.-Ing. S. Berz, HRSflow GmbH, Frankfurt/M.

Ausschussreduktion durch automatisierte Datenanalyse - Anomalieerkennung von Prozessdaten

Dr. M. Wank, Dr.-Ing. S. Kruppa, M. Busl, KraussMaffei Technologies GmbH, München

Energieeffiziente Temperierung - Sind Klimaschutz und wirtschaftliche Kaufinteressen vereinbar?

R. Radke, gwk Gesellschaft Wärme Kältetechnik mbH, Meinerzhagen

Stellschrauben für die Zykluszeitoptimierung am Beispiel der

Pflanztopffertigung

Dipl.-Ing. (FH) G. Orschulik, Pöppelmann, Lohne 